\title{
Ventilator-Associated Events : définition et intérêts ?
}

\author{
Ventilator-Associated Events: Definition and Interests?
}

\author{
M. Neuville $\cdot$ L. Bouadma $\cdot$ A. Radjou $\cdot$ E. Magalhaes $\cdot$ R. Smonig $\cdot$ G. Voiriot $\cdot$ J.-F. Soubirou $\cdot$ R. Sonneville $\cdot$ \\ B. Mourvillier $\cdot$ J.-F. Timsit
}

Reçu le 21 septembre 2015 ; accepté le 6 octobre 2015

(C) SRLF et Lavoisier SAS 2015

Les pneumonies associées à la ventilation mécanique (PAVM) sont une complication majeure de la ventilation mécanique et un marqueur reconnu de la qualité des soins d'un service de réanimation [1]. La création et la mise en place de stratégies d'intervention associant plusieurs mesures de prévention sous forme de bundle ont entraîné une baisse significative de ces infections au cours des dix dernières années [2]. Les progrès ont été tels que certains ont cru possible d'atteindre l'objectif de «zéro PAVM », rendant ainsi toute infection acquise sous ventilation mécanique potentiellement équivalente à une erreur médicale [3].

À dire vrai, ce concept de « zéro PAVM » semble plutôt irréaliste, à moins qu'il ne résulte de l'utilisation de définitions imparfaites de la PAVM ? En effet, il n'existe pas actuellement de consensus concernant les critères diagnostiques de PAVM, ce qui laisse une place majeure à l'interprétation et à la subjectivité, notamment dans l'analyse des données cliniques ou des infiltrats radiologiques. Des travaux belges ont récemment trouvé en appliquant rétrospectivement des critères diagnostiques différents un taux de survenue de PAVM allant de 0 à $44 \%$ dans une même population de patients [4].

Dans ce contexte, le Center for Disease Control and Prevention (CDC) a donc proposé un nouveau concept associant des critères objectifs pour tenter de mieux surveiller la survenue d'infections acquises sous ventilation mécanique, les événements associés à la ventilation (ventilator-

\footnotetext{
M. Neuville $\cdot$ L. Bouadma $\cdot$ A. Radjou $\cdot$ E. Magalhaes $\cdot$ R. Smonig $\cdot$ G. Voiriot $\cdot$ J.-F. Soubirou $\cdot$ R. Sonneville B. Mourvillier · J.-F. Timsit $(\bowtie)$

Assistance Publique Hôpitaux de Paris / hôpital Bichat réanimation médicale et des maladies infectieuses, 46 rue Henri Huchard, F-75018Paris

e-mail : Jean-francois.timsit@bch.aphp.fr
}

L. Bouadma $\cdot$ B. Mourvillier $\cdot$ J.-F. Timsit

UMR 1137 - IAME Equipe5 - DeSCID (Decision SCiences in Infectious Diseases), control and care, Inserm/Univ Paris Diderot, Sorbonne Paris Cité, F-75018 Paris, France associated events [VAE]) [5]. Ceux-ci sont au nombre de trois : la ventilator-associated condition (VAC) correspond à la détérioration de l'état respiratoire du patient après une période de stabilité ou d'amélioration de deux jours, objectivée par la nécessité d'augmenter la pression expiratoire positive (PEEP) ou la $\mathrm{FiO}_{2}$ de façon prolongée (plus de 48h). En cas de signes d'inflammation ou d'infection (fièvre ou hypothermie, tachycardie, leucopénie ou hyperleucocytose) et de la prescription d'un nouvel anti-infectieux poursuivie pendant au moins quatre jours, la VAC devient une IVAC, soit une infection-related ventilator-associed complication. Enfin, si l'on documente la présence d'un agent infectieux sur des prélèvements pulmonaires ou pleuraux, la PAVM devient possible, ce qui est exprimé par le sigle PVAP, ou possible ventilator-associated pneumonia.

Les avantages supposés de cet algorithme diagnostique sont notamment l'absence d'interprétation de la radiographie thoracique et la possibilité de surveiller la survenue de ces événements de façon automatisée en enregistrant par exemple les paramètres de réglage du ventilateur et les constantes du patient. Cependant, l'utilisation de ce triple concept VACIVAC-PVAP dans la pratique clinique n'a pas été validée. Les études sont d'ailleurs discordantes lorsqu'il s'agit d'évaluer le taux de prévalence de ces trois événements dans un service de réanimation. Une étude américaine publiée en 2011 [6] trouvait qu'environ $40 \%$ des patients chez qui l'on diagnostiquait une VAC avaient finalement une PAVM. Dans une étude multicentrique sur la base de données Outcomerea, nous avons montré que seuls $15 \%$ des VACs et $28 \%$ des IVACs étaient liés à une PAVM chez les patients ventilés plus de cinq jours [7]. A contrario, des travaux canadiens [8] montraient une prévalence équivalente des VACs $(10,5 \%)$ et des VAPs $(11,2 \%)$. Il est même possible qu'une VAP ne remplisse pas tous les critères de VAC, comme l'illustre la publication récente de Boyer et al., où seuls 21 épisodes de VAP sur les 86 rapportés pouvaient être qualifiés d'IVAC [9].

Les publications ayant étudié l'association des VAEs à la mortalité ne sont pas non plus unanimes. Alors que la 
survenue d'une VAC semble associée à un taux de mortalité plus important dans certains travaux récents, notamment ceux de Boyer et al. (66 vs $14 \%$ ) [9], d'autres trouvent des taux identiques, en présence ou non de VACs [10]. On note cependant de façon plus homogène une durée de ventilation mécanique plus importante lorsqu'une VAC est diagnostiquée au cours du séjour [7].

La définition des VAE laisse apparaître des faiblesses, qui peuvent expliquer en partie les discordances observées en termes de prévalence et d'impact pronostique. En effet, les critères diagnostiques de VAC ne permettent d'en poser le diagnostic qu'après au minimum quatre jours de ventilation mécanique, ce qui exclut l'inclusion de patients décédés précocement. Les variations de $\mathrm{PEEP}$ et de $\mathrm{FiO}_{2}$ sont laissées à la discrétion du clinicien et non basées sur des critères gazométriques, ce qui laisse libre cours à toutes les pratiques. Certes, l'enregistrement automatisé des paramètres du ventilateur facilite le recueil des données. Il paraît tout de même assez aisé de réduire de façon drastique le taux de prévalence des VACs en gardant une PEEP ou une $\mathrm{FiO}_{2}$ élevée de façon non nécessaire, afin de prévenir la survenue d'une dégradation de l'état respiratoire du malade.

Les taux de VAC et IVAC sont corrélés à la consommation d'antibiotiques dans un service de réanimation $[7,10]$. Cependant, l'attrait des VAC comme outil de benchmarking pour comparer les unités entre elles doit être modulé par la faible valeur de la VAC dans le diagnostic de PAVM. Des études complémentaires devraient démontrer qu'une baisse des VAC est associée à une baisse des consommations d'antibiotiques et que le rapport IVAC/consommation d'antibiotiques est un bon indicateur de processus de soins.

Les premières études disponibles ne plaident malheureusement pas en ce sens. Dans le travail de Muscedere et al., l'application d'un bundle de prévention des PAVM a permis de réduire les VACs, mais pas les IVACs [8]. De même, une étude comparant la survenue de PAVM avec et sans système d'aspiration sus-glottique associé à la sonde d'intubation montrait une réduction significative de la prévalence des PAVM et de la consommation d'antibiotiques dans le groupe possédant ce système, alors que le taux de VACs était identique dans les deux groupes [11].
Aussi, à l'heure actuelle, bien que simples à recueillir, il semble difficile d'utiliser les VACs comme indicateurs de la qualité des soins d'un service de réanimation, principalement parce que la plupart des VACs ne sont pas des PAVM et que nous ne disposons pas de moyen de prévention de ces événements, qu'ils soient des VAC ou des IVACs.

\section{Références}

1. Bouadma L, Wolff M, Lucet JC (2012) Ventilator-associated pneumonia and its prevention. Curr Opin Infect Dis 25:395-404

2. Bouadma L, Deslandes E, Lolom I, et al (2010) Long-term impact of a multifaceted prevention program on ventilatorassociated pneumonia in a medical intensive care unit. Clin Infect Dis Off Publ Infect Dis Soc Am 51:1115-22

3. Klompas M (2012) Is a ventilator-associated pneumonia rate of zero really possible? Curr Opin Infect Dis 25:176-82

4. Ego A, Preiser JC, Vincent JL (2015) Impact of diagnostic criteria on the incidence of ventilator-associated pneumonia. Chest 147:347-55

5. ACH Surveillance for VAE | NHSN | CDC n.d. http://www.cdc. gov/nhsn/acute-care-hospital/vae/ (accessed July 29, 2015)

6. Magill SS, Klompas M, Balk R, et al (2013) Developing a new, national approach to surveillance for ventilator-associated events: executive summary. Clin Infect Dis Off Publ Infect Dis Soc Am 57:1742-6

7. Bouadma L, Sonneville R, Garrouste-Orgeas M, et al (2015) Ventilator-Associated Events: Prevalence, Outcome, and Relationship With Ventilator-Associated Pneumonia. Crit Care Med 43:1798-806

8. Muscedere J, Sinuff T, Heyland DK, et al (2013) The clinical impact and preventability of ventilator-associated conditions in critically ill patients who are mechanically ventilated. Chest 144:1453-60

9. Boyer AF, Schoenberg N, Babcock H, et al (2015) A prospective evaluation of ventilator-associated conditions and infectionrelated ventilator-associated conditions. Chest 147:68-81

10. Hayashi Y, Morisawa K, Klompas M, et al (2013) Toward improved surveillance: the impact of ventilator-associated complications on length of stay and antibiotic use in patients in intensive care units. Clin Infect Dis Off Publ Infect Dis Soc Am 56:471-7

11. Damas P, Frippiat F, Ancion A, et al (2015) Prevention of ventilator-associated pneumonia and ventilator-associated conditions: a randomized controlled trial with subglottic secretion suctioning. Crit Care Med 43:22-30 